\title{
A Network Pharmacology Approach and Molecular Docking Technology to Uncover the Potential Molecular Mechanism of Astragalus -Scorpion on Prostate Cancer
}

\section{Litong Wu}

Guangzhou University of Traditional Chinese Medicine: Guangzhou University of Chinese Medicine https://orcid.org/0000-0002-3023-0486

\section{Ying Chen}

Guangzhou University of Traditional Chinese Medicine: Guangzhou University of Chinese Medicine Minjing Chen

Guangzhou University of Traditional Chinese Medicine: Guangzhou University of Chinese Medicine

\section{Yueqin Yang}

Guangzhou University of Traditional Chinese Medicine: Guangzhou University of Chinese Medicine

\section{Zuzhao Che}

Guangzhou University of Traditional Chinese Medicine: Guangzhou University of Chinese Medicine

\section{Qixin Li}

Guangzhou University of Traditional Chinese Medicine: Guangzhou University of Chinese Medicine

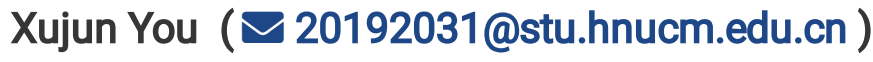

Guangzhou University of Traditional Chinese Medicine: Guangzhou University of Chinese Medicine https://orcid.org/0000-0002-6118-2309

\section{Wei Fu}

Guangzhou University of Traditional Chinese Medicine: Guangzhou University of Chinese Medicine

\section{Research}

Keywords: Prostate cancer, Network pharmacology, Astragalus, Scorpion, Molecular docking

Posted Date: February 15th, 2021

DOl: https://doi.org/10.21203/rs.3.rs-203879/v1

License: (c) (i) This work is licensed under a Creative Commons Attribution 4.0 International License. Read Full License 


\section{Abstract}

Objective: To investigate the molecular mechanism of Astragalus-Scorpion in the treatment of prostate cancer by network pharmacology and molecular docking technology.

Methods: Using TCMSP, BATMAN-TCM, TCMID and Swiss TargetPrediction Databases to retrieve the active ingredients and corresponding targets of Astragalus-Scorpion. The targets related to prostate cancer were retrieved through GeneCards, so as to obtain the common targets of Astragalus-Scorpion and prostate cancer. The common targets were input into the STRING database for protein interaction analysis. Cytoscape software was used to construct the active "ingredient-target-disease" network, and GO and KEGG enrichment analysis were performed on the common targets. To screen the core ingredients and targets that play therapeutic roles, Autodock software was used for molecular docking verification.

Results: 27 active ingredients, 340 potential targets related to active ingredients, 898 targets related to disease and 122 common targets were screened from Astragalus-Scorpion totally. The core targets of PPI network were JUN, AKT1, IL6, MAPK1 and RELA, while the core active ingredients in the active ingredienttarget-disease network were quercetin, kaempferol, formononetin, 7-o-methylisomucronulatol and calycosin. $762 \mathrm{GO}$ entries and 154 pathways were obtained by enrichment analysis totally. The molecular docking results showed that quercetin binds to AKT1 and RELA, kaempferol to AKT1, and formononetin to RELA, all of which were stable.

Conclusion: Quercetin, kaempferol and others in the Astragalus-Scorpion can regulate multiple signaling pathways such as PI3K-Akt signaling pathway by binding to targets such as AKT1 related to prostate cancer, inhibit the proliferation of tumor to play a role for prostate cancer.

\section{Introduction}

Prostate Cancer ( $\mathrm{PCa}$ ) ranks second in the incidence of cancer in middle-aged and elderly men, and its mortality rate ranks fifth in the world of cancer mortality [1]. In recent years, the incidence of PCa in Asian countries continues to increase [2]. Recent studies have shown that the incidence and mortality of PCa in China are also increasing year by year [3]. PCa is highly invasive and a serious threat to the life of men. Currently, the clinical treatment of prostate cancer is still dominated by androgen blockade, supplemented by radiotherapy and chemotherapy. Androgen deprivation therapy has been the most effective and widely used treatment for prostate cancer in the past 70 years [4]. However, regardless of surgical castration or drug castration, anti-androgen therapy and chemotherapy will show treatment resistance, and patients with advanced castration resistant prostate face no medicine to treat [5]. Although some progress has been made in new anti-androgen drugs, chemotherapy, palliative radiotherapy, radionuclide therapy and immunotherapy, PCa treatment is still a major clinical problem. The search for a natural drug with high effect and low toxicity for the prevention and treatment of prostate cancer has become a current research hotspot [6,7]. Studies have found that traditional Chinese medicine contains a variety of ingredients, has 
the advantages of multi-target, multi-function and synergistic effect, effectively prevents the proliferation and metastasis of tumor cells, and has a good prospect of application [8].

Astragalus with sweet taste in five flavors and mild temperature in four Qi attributes to lung and spleen meridians. with the effects of tonifying Qi, raising Yang, removing stasis, relieving obstruction and detoxifying. Scorpion with flat in four Qi, it belongs to liver meridian, has the effect of detoxification, dissipation of nodules, inhibition of cancer, pain relief. Astragalus-Scorpion drug pair are commonly used in TCM treatment of tumors. Astragalus membranaceus is good at benefiting Qi and strengthening the body, while Scorpion is more prone to detoxify and inhibit cancer. The two drugs are compatible with each other, one defending and one attacking, complementing each other and synergistic effect, so as to achieve the effect of strengthening Qi and inhibiting cancer.

However, the pharmacodynamics study of Astragalus-Scorpion drug pair in the treatment of PCa is still in the basic stage, and there are few studies on its target and molecular mechanism. Therefore, based on the research method of network pharmacology [9], this paper studies the potential molecular mechanism of Astragalus-Scorpion drug pair in the treatment of $\mathrm{PCa}$, and carries out molecular docking technology to verify the docking of core active ingredients related to Astragalus-Scorpion drug pair and core targets related to PCa, providing scientific basis for further elucidating the mechanism at the molecular level.

\section{Materials And Methods}

\subsection{Extraction and screening of active ingredients of Astragalus - Scorpion drug pair}

Oral Bioavailability $(\mathrm{OB})>30 \%$ and Drug Likeness $(\mathrm{DL})>0.18$ were set up in the Traditional Chinese Medicine Systems Pharmacology (TCMSP,https://tcmspw.com/tcmsp.php) [10]. Score cutoff $>20$ and $p<0.05$ were set up in the Bioinformatics Analysis Tool for Molecular mechanism of Traditional Chinese Medicine [11] هBATMAN-TCM, http://bionet.ncpsb.org/batman-tcm/囚, while Astragalus and Scorpion were input into Traditional Chinese Medicine Integrated Database (TCMID,http://119.3.41.228:8000/tcmid/拜 The potential active ingredients of Astragalus-Scorpion were screened from 3 databases mentioned above, and to ensure scientific nature and integrity of the retrieval by combining with literature retrospective investigation and verification.

\section{Figure 1. Network pharmacology analysis of Astragalus- Scorpion drug pair on prostate cancer}

\subsection{Collection of targets related to Astragalus-Scorpion drug pair}


The active ingredients collected above were input into TCMSP, BATMAN-TCM and Swiss Target Prediction(http://www.swisstargetprediction.ch/) respectively to obtain corresponding targets. After removing the repetitive part, the targets were imported into the UniProt (https://www.uniprot.org/) . The protein species of target were set to "Homo sapiens" and the target source was set to be "reviewed". A normalized Gene Symbol for subsequent network analysis was obtained after gene name matching and standardization.

\subsection{Acquisition and collection of targets related to prostate cancer}

Genecards (https://www.genecards.org/) is a gene-centricity human genome database [12], automatically integrating sources from 150 websites. The targets of PCa can be collected by setting the search words as "prostate cancer" and "Relevance Score" $\geq 10$.

\subsection{Acquisition of the common targets of Astragalus- Scorpion drug pair and prostate cancer}

We input standardized targets of Astragalus-Scorpion and targets related to PCa into Venny (ver. 2.1.0) (https://bioinfogp.cnb.csic.es/tools/venny/) and made Venny diagram to obtain the common targets.

\subsection{Construction and analysis of protein-protein interaction (PPI) network}

The STRING [13] (https://string-db.org) is a biological database for predicting protein-protein interactions. The common targets mentioned above were uploaded to the STRING(https://string-db.org) in the form of gene symbol. PPI network interaction diagram was obtained by setting the species as "homo sapiens ",the minimum interaction threshold as "high confidence $\triangle 0.700 \rrbracket$ " and leaving the other settings on the default parameters. The count.R plug-in in the R language software (https://www.r-project.org/) was used to obtain the frequency of the common protein targets and further to screen the core targets.

\subsection{Network construction of active ingredient-target- disease}

The active ingredient-target-disease network was constructed by using the MERGE function of Cytoscape (ver. 3.7.1) [14]. In the network diagram, "Node" represents drugs, active ingredients, diseases and targets, while "edge" represents the relationship among active ingredients of drugs, targets and prostate cancer. The potential mechanism of Astragalus - Scorpion in the treatment of PCa was explored based on the network constructed above. 


\subsection{GO analysis and KEGG pathway enrichment analysis}

In order to further reflect the functions of the common targets screened above and their roles in the signaling pathways, gene ontology analysis (Go analysis) and Kyoto Encyclopedia of Genes and Genomes (KEGG) pathway analysis of common targets mentioned above were performed using the cluster ProfilerGo.R plug-in for R software and Perl language. The GO enrichment analysis consists of biological processes (BP), cellular components (CC) and molecular functions (MF). The top 45 potential

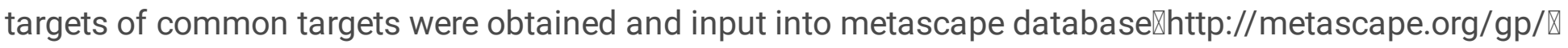
for enrichment analysis. Then the results were input into bioinformatics database \http://www.bioinformatics.com.cn/囚to obtain KEGG enrichment string-figure.

\subsection{Molecular docking of main potential active ingredients and targets}

In order to further verify the reliability of targets predicted, molecular docking verification was conducted among the top 5 core active ingredients screened and the top 5 core targets. First of all】the threedimensional (3D) structure of the core protein was downloaded from the PDB database (https://www.rcsb.org/), imported into PyMoL (ver. 2.3.1) to remove water molecules and small molecules of acceptors $囚$ imported into Autodock Tools (ver. 1.5.6) [15] to add hydrogen atoms, calculate the total charge and set the atomic type and then saved to pdbqt format. Secondly, mol 2 format of the core active ingredients (ligand) screened above was downloaded from the TCMSP and saved as "PDBQT" format after selecting "Check totals on residues", "detect root" and "choose torsions" keys in turn. Finally, Autogrid4 and Autodock4 were used for space positioning and molecular docking. "Local Search Parameters" algorithm was used for the docking operation and "Live Parameters" was used as the docking parameter. We use PyMoL software to realize the visualization of docking results and establish the docking interaction pattern diagram.

\section{Results}

\subsection{Screening of Astragalus - Scorpion active ingredients}

A total of 20 active ingredients of Astragalus and a number of 7 active ingredients of Scorpion were obtained by using TCMSP, BATMAN-TCM, TCMID and combining with literature retrospective investigation to verify and supply. The screening results of effective ingredients of astragalus are shown in Table 1. 
Table 1 Main active ingredients of Astragalus - Scorpion

\begin{tabular}{|c|c|c|c|}
\hline $\begin{array}{l}\text { Ingredient } \\
\text { number }\end{array}$ & Ingredient name & $\mathrm{OB} \%$ & DL \\
\hline MOL000387 & Bifendate & 31.1 & 0.67 \\
\hline MOL000033 & $\begin{array}{l}\text { (3S,8S,9S,10R,13R,14S,17R)-10,13-dimethyl-17-[(2R,5S)-5-propan-2- } \\
\text { yloctan-2-yl]-2,3,4,7,8,9,11,12,14,15,16,17-dodecahydro-1H- } \\
\text { cyclopenta[a]phenanthren-3-ol }\end{array}$ & 36.23 & 0.78 \\
\hline MOL000379 & 9,10-dimethoxypterocarpan-3-O- $\beta$-D-glucoside & 36.74 & 0.92 \\
\hline MOL000296 & hederagenin & 36.91 & 0.75 \\
\hline MOL000442 & 1,7-Dihydroxy-3,9-dimethoxy pterocarpene & 39.05 & 0.48 \\
\hline MOL000374 & 5'-hydroxyiso-muronulatol-2',5'-di-O-glucoside & 41.72 & 0.69 \\
\hline MOL000422 & kaempferol & 41.88 & 0.24 \\
\hline MOL000098 & quercetin & 46.43 & 0.28 \\
\hline MOL000417 & Calycosin & 47.75 & 0.24 \\
\hline MOL000439 & isomucronulatol-7,2'-di-O-glucosiole & 49.28 & 0.62 \\
\hline MOL000354 & isorhamnetin & 49.6 & 0.31 \\
\hline MOL000239 & Jaranol & 50.83 & 0.29 \\
\hline MOL000371 & 3,9-di-O-methylnissolin & 53.74 & 0.48 \\
\hline MOL000211 & Mairin & 55.38 & 0.78 \\
\hline MOL000380 & $\begin{array}{l}\text { (6aR,11aR)-9,10-dimethoxy-6a,11a-dihydro-6H-benzofurano[3,2- } \\
\text { c]chromen-3-ol }\end{array}$ & 64.26 & 0.42 \\
\hline MOL000438 & (3R)-3-(2-hydroxy-3,4-dimethoxyphenyl)chroman-7-ol & 67.67 & 0.26 \\
\hline MOL000433 & FA & 68.96 & 0.71 \\
\hline MOL000392 & formononetin & 69.67 & 0.21 \\
\hline MOL000378 & 7-0-methylisomucronulatol & 74.69 & 0.3 \\
\hline MOL000398 & isoflavanone & 109.99 & 0.3 \\
\hline
\end{tabular}

\subsection{Prediction and screening of targets}


Through the prediction and selection of TCMSP,BATMAN-TCM and Swiss TargetPrediction database, after standardized treatment of UniProt database, a total of 180 potential targets of active ingredients of Astragalus and 176 of Scorpion were obtained. And a total of 898 potential targets associated with PCa were obtained through the prediction and screening of GeneCards database. Then, we acquired 340 potential targets of Astragalus and Scorpion after removing repetitive targets. Further, 122 common targets were acquired after importing the above-mentioned targets and the targets related to PCa into Venny database, as shown in Figure 2a.

\subsection{Construction and analysis of protein-protein interaction (PPI) network}

A total of 122 targets obtained above were input into STRING database to obtain protein interaction relationship, as shown in Figure $2 \mathrm{~b}$. The count.R plug-in was used to count the frequency of protein target interconnection, and the top 30 proteins were selected for visual display, as shown in Figure 2c. The top 5 core proteins were JUN, AKT1, IL6, MAPK1 and RELA, which may be beneficial to Astragalus-Scorpion drug pair in the treatment of PCa.

\subsection{Active ingredient - target - disease network}

Cytoscape(ver. 3.7.1) was used to construct the network of drug pair active ingredient-common targetPCa, and a network with 144 nodes and 373 edges was obtained, as shown in Figure 3 . The hexagon represents $\mathrm{PCa}$, the quadrilateral Astragalus-Scorpion drug pair, the triangle the active ingredients, and the oval the common target. The connections between the points indicate the targeting relationship among the active ingredient, the target and the disease. The more connections there are, the more important the nodes is in the network. The top 5 active ingredients in the number of connections are quercetin, kaempferol, formononetin, 7-0-methylisomucronulatol and Calycisin, which may play an important role in the treatment of prostate cancer.

\subsection{GO enrichment analysis and KEGG pathway enrichment analysis}

Cluster Profiler GO.R plug-in and Perl language were used to conduct bioinformatics analysis of the above 122 common targets. In this enrichment analysis, a total of $762 \mathrm{GO}$ entries were obtained. There were 50 cell components (CC), which had significant effects on cell components such as Cytosol, Nucleus, Mitochondria, Membrane and extracellular matrix. There were 611 biological processes (BP), mainly involving positive regulation of transcription from RNA polymerase II promoter, negative regulation of apoptotic process, positive regulation of transcription, DNA-templated and response to drug, which showed that the Astragalus-Scorpion drug pair can play an anti-cancer effect by participating in the regulation of various biological processes. There were 101 Molecular Function, mainly including protein binding, enzyme binding, DNA binding and tumor necrosis factor receptor binding. Among them, the top 10 entries respectively in BP, CC and MF were visually analyzed, as shown in Figure 4. A total of 154 pathways of common targets were obtained by KEGG signaling pathways enrichment. The top 20 pathways were screened out $(P<0.01)$, as shown in Figure $5 a$ and Figure $5 b$. The main signaling pathways contained PI3K-Akt signaling pathway, AGE-RAGE signaling pathway, TNF signaling pathway, 
IL-17 signaling pathway and so on, suggesting that Astragalus-Scorpion can treat PCa through multiple signaling pathways. Five signal pathways with high correlation were displayed in the KEGG enriched string diagram, as shown in Figure 6. Taking the PI3K-Akt signaling pathway for example, the potential target and mechanism of Astragalus-Scorpion drug pair in the treatment of PCa were shown in Figure 7.

\subsection{Molecular docking verification}

Molecular docking was carried out for the 5 core active components and 5 core targets screened from Astragalus-Scorpion drug pair. The basic information of the protein and the original ligand corresponding to the target were shown in Table 2. The binding energy and the number of hydrogen bonds obtained by molecular docking were shown in Table 3 and Table 4. From the docking results $邓$ the binding energy values of quercetin and AKT1, quercetin and RELA, formononetin and RELA, and kaempferol and AKT1 were all less than $-5 \mathrm{kcal} / \mathrm{mol}$ 区indicating that the three active ingredients had strong binding force with the two core targets. The number of hydrogen bonds of quercetin and RELA, quercetin and AKT1, as well as kaempferol and AKT1 were large, which may have good stability. Based on the above results, it showed that quercetin, formononetin and kaempferol may play an important role in the treatment of PCa. The molecular docking model diagrams of quercetin and AKT1, quercetin and RELA, formononetin and RELA, as well as kaempferol and AKT1 were selected respectively for visual display, as shown in Figure 8.

Table 2 Information of 5 proteins involved in molecular docking

\begin{tabular}{|llll|}
\hline targets & Uniprot-ID & PDB-ID & Ligand-ID \\
\hline JUN & P05412 & 5FV8 & JEF \\
\hline AKT1 & P31749 & 3096 & IQO \\
\hline IL6 & P05231 & 1ALU & TLA \\
\hline MAPK1 & P28482 & 1TVO & FRZ \\
\hline RELA & Q04206 & 6QHL & SEP \\
\hline
\end{tabular}

Table 3 The binding energy of Astragalus-Scorpion core active ingredients and core common targets 


\begin{tabular}{|llllll|}
\hline Core active ingredient & \multicolumn{5}{c|}{ Binding Energy $/\left(\mathrm{kcal}^{\prime} \mathrm{mol}^{-1}\right)$} \\
\cline { 2 - 6 } & JUN & AKT1 & IL6 & MAPK1 & RELA \\
\hline quercetin & -3.59 & -5.36 & -3.61 & -5.83 & -8.20 \\
\hline kaempferol & -4.51 & -5.41 & -2.99 & -3.11 & -3.22 \\
\hline formononetin & -3.83 & -4.50 & -4.21 & -4.27 & -7.90 \\
\hline 7-O-methylisomucronulatol & -4.32 & -3.42 & -3.21 & -3.32 & -3.53 \\
\hline Calycisin & -2.67 & -3.30 & -3.33 & -3.50 & -2.87 \\
\hline
\end{tabular}

Table 4 The number of hydrogen bonds of Astragalus-Scorpion core active ingredients and core common targets

\begin{tabular}{|llllll|}
\hline Core active ingredient & \multicolumn{5}{l|}{ The number of hydrogen bonds } \\
\cline { 2 - 6 } & JUN & AKT1 & IL6 & MAPK1 & RELA \\
quercetin & 2 & 5 & 4 & 1 & 4 \\
\hline kaempferol & 3 & 5 & 3 & 3 & 4 \\
formononetin & 2 & 3 & 3 & 3 & 2 \\
7-O-methylisomucronulatol & 4 & 1 & 1 & 2 & 0 \\
Calycisin & 0 & 2 & 3 & 4 & 1 \\
\hline
\end{tabular}

\section{Discussion}

Based on the research method of network pharmacology, this study finally predicted 27 active ingredients, 122 potential targets, 762 Go analysis and 154 KEGG pathways of Astragalus-Scorpion drug pair in the treatment of $\mathrm{PCa}$. The interaction of multi-ingredient, multi-target and multi-pathway further demonstrated the principle of traditional Chinese medicine treatment of PCa from the pharmacological and molecular levels: consolidate the root, eliminate the source of disease, strengthening the body, eliminate pathogen.

Modern pharmacological studies have confirmed that the chemical ingredients of Astragalus-Scorpion drug pair are mainly flavonoids, saponins and polysaccharides [16], which can improve immunity, promote metabolism, regulate blood glucose, improve the function of the heart, inhibit virus, and have anti-tumor effect [17].The chemical ingredients of Scorpion are mainly Scorpion venom, steroid 
derivatives, alkaloids and other small molecular ingredients. The enzymatic hydrolysate of Scorpion venom can inhibit the growth of tumor cells and induce tumor cell apoptosis to achieve anti-tumor effect [18]. In this study, most of the ingredients screened by the Astragalus-Scorpion drug pair were flavonoids, such as quercetin, kaempferol, formononetin, calycosin and so on. Relevant studies have found that quercetin can promote AGS cell apoptosis by increasing the production of reactive oxygen species (ROS), reduce the expression of anti-apoptotic protein and increase the expression of pro-apoptotic protein [19]. In addition, quercetin can reduce the expression of genes associated with the apoptotic pathway, such as VEGFB. Abdur Rauf et al. [20] also showed that quercetin has inhibitory effect on a variety of cancers, high toxic effect on tumor cells, and less toxic and side effects on normal tissue cells, indicating that quercetin has a high targeting effect on killing tumor cells. Kaempferol has been used for cancer chemotherapy, which has many pharmacological properties, such as anti-bacteria, anti-inflammation, anti-oxidation, anti-tumor, cardiac-protection, neuro-protection and anti-diabete. Its mechanisms of anticancer include induction of apoptosis, G2/M-phase cell cycle arrest, down-regulation of markers related to epithelial mesenchymal transformation (EMT), and phosphoinositi-3kinase/protein kinase B signaling pathways [21]. Relevant studies have shown that formononetin can play an anti-tumor effect by regulating signal pathways such as JAK-STAM [22], and the expression of caspase, 1VIIVIPS, GSK-3P, Axin and other molecules in vivo and in vitro [23-26]. It is reported that Formononetin can inhibit the proliferation of PCa cells mainly through MAPK, PI3K / Akt and IGF-1 / IGF-1R pathways. Calycosin can improve immune function, enhance the effect of anti-oxidation, anti-radiation and anti-cancer, and has the function of lowering blood fat, blood sugar and reducing complications of diabetes. Its anti-tumor effect is mainly to increase the expression level of MMP9 and P-Akt by expressing C-Metu87》and further to prevent the invasion of cancer cells [27]. In conclusion, the main active ingredients of AstragalusScorpion drug pair in the treatment of Pca were collected comprehensively in this study.

The common targets (JUN, AKT1, IL6, MAPK1 and RELA)are at the core of PPI interaction network, which may play an important role in the treatment of prostate cancer with Astragalus-Scorpion drug pair. JUN is the first found carcinogenic transcription factor. C-JUN is an AR co-activator, which stimulates AR transactivation by mediating receptor dimerization and subsequent DNA binding, and plays a key role in the proliferative function of fibroblasts and the anti-proliferative activity of finasteride on epithelial cells [28]. IL6, a glycogen composed of 184 amino acids, is a pluripotent cytokine that affects tumor cell activity and plays an important role in immune response and inflammation [29,30]. Studies have shown that IL6 can interfere inflammatory environment, induce tumor occurrence, carry out microenvironment immune regulation and induce apoptosis of cancer cells [31-33]. These effects are performed through a variety of pathways, among which signal transducers and activators of transcription play the most important roles [34]. In addition, studies have shown that IL6 is overexpressed in a variety of cancer cells, and it is significantly increased in advanced cancer [33,35]. MAPK signaling pathway plays a key role in various cancers through hyperactivation and is an important part of cancer pathway. MAPK can transfer extracellular signals into various cellular processes, such as cell proliferation, cell survival, cell death and cell differentiation $[36,37]$. The current evidence clearly shows that MAPK pathway is a feasible target for cancer treatment [38]. AKT1 is an important gene involved in tumor invasion and angiogenesis. AKT1 is 
the core target of $\mathrm{PI} 3 \mathrm{~K} / \mathrm{Akt}$ signaling pathway, which plays a role in regulating cell proliferation, cell apoptosis, cell cycle, cell metabolism, protein transcription and angiogenesis. Abnormal activation of Akt can avoid apoptosis and promote cell survival, which is related to the occurrence of tumor $[39,40]$. According to current research, exogenous AKT1 promotes the migration and invasion of breast cancer cell. The growth and invasion of breast cancer cells can be inhibited by specifically inhibiting the expression of AKT1 [41]. RELA, a kind of NF-KB, can induce the expression of various proteins involved in inflammation and carcinogenesis, such as FAS, cyclin, IL-2, tumor necrosis factor(TNF), CXCL1 and matrix metallopeptidase (MMPs), which can promote the survival and proliferation of tumor cells, inflammatory response, anti-apoptosis, angiogenesis and metastasis [42-44]. Studies have shown that the abnormal activation of NF-KB / RELA and the imbalance of microRNA are related to the cell proliferation of non-small cell lung cancer. MicroRNA affects cell proliferation of non-small cell lung cancer by degrading RELA or inhibiting its expression. For example, mir506 selectively eliminates lung cancer cells by inhibiting the expression of RELA, producing reactive oxygen species and activating p53 [45].

In this study, by inputting common targets for GO analysis and KEGG analysis, it was found that targets of Astragalus-Scorpion drug pair for PCa mainly concentrated in cancer-related pathways, PI3K-Akt signaling pathway, AGE-RAGE signaling pathway, TNF signaling pathway and so on. GO analysis mainly involves transcription from RNA polymerase II promoter, apoptotic process, DNA transcription and other biological processes. The active ingredients of Astragalus-Scorpion promote the apoptosis of PCa cells by inhibiting the cell proliferation and cell differentiation, and is related to the biological regulation and biological process of tumor cells. PI3K-Akt signaling pathway is one of the most classic tumor survival pathways, which plays an important role in the proliferation and differentiation of tumor cells $[46,47]$. Fizazi et al. [48] found that inhibition of PI3K-Akt signaling pathway can effectively prevent the proliferation and differentiation of prostate cancer cells. Yan et al. [49] believed that activation of PI3KAkt signaling pathway would inhibit the transcription of FOXO, a subgroup of downstream apoptotic related gene forkhead box transcription factor, and increase the level of FOXO phosphorylation, so as to block the apoptosis of PCa cells. AGE is the product of the Maillard reaction between sugars and proteins in human body. RAGE, a membrane protein belonging to the immunoglobulin superfamily, is named because it acts as the receptor of AGE and interacts with it to produce biological effects [50,51]. AGERAGE signaling pathway plays an important role in the occurrence and development of prostate cancer [52]. Elangovan et al. [53] found that specific blocking RAGE and its downstream targets can induce apoptosis and inhibit proliferation of prostate cancer cells in vivo and in vitro. In addition, relevant studies have shown that the activation of AGE-RAGE signaling pathway can further activate PI3K-Akt signaling pathway to promote the proliferation of prostate cancer cells [54].

According to the results of molecular docking, the binding energies between the core active ingredient and the core target are all less than $0 \mathrm{kcal} / \mathrm{mol}$, indicating that there is a possibility of binding between the active ingredient and the target. The binding energy less than or equal to $-5.0 \mathrm{kcal} / \mathrm{mol}$ is considered to have good binding ability [55]. The binding energies of quercetin with AKT1, quercetin with RELA and kaempferol with AKT1 were all less than $-5.0 \mathrm{kcal} / \mathrm{mol}$, and the number of hydrogen bonds was more 
than or equal to 4, indicating that their binding ability was strong and stable. Quercetin and kaempferol, the active ingredients, selectively inhibit the activation of PI3K-Akt signaling pathway by binding to AKT1, a key target in the PI3K-Akt signaling pathway, so as to inhibit the proliferation of prostate cancer cells. This may be one of the important molecular mechanisms of Astragalus-Scorpion drug pair in the treatment of PCa in this study.

\section{Conclusiion}

TCM treatment of PCa has the advantages of multi-component, multi-target, light toxic and side effects, preventing recurrence and metastasis after operation and so on. As a typical treatment for PCa, the potential mechanism of Astragalus-Scorpion drug pair needs further study. In this study, the network pharmacology was used for the first time to screen out the potential active ingredients of AstragalusScorpion drug pair, obtain the potential targets for the treatment of $\mathrm{PCa}$, construct the network of "drugactive ingredient-target-disease", and further verify the molecular mechanism of Astragalus-Scorpion drug in the treatment of PCa. In addition, quercetin, kaempferol, formononetin, 7-0-Methylisomucronulato and calycosin are key active ingredients of Astragalus-Scorpion drug pair in the treatment of PCa. Jun, Akt1, IL6, MAPK1 and RelA are key genes that play roles in signaling pathways such as PI3K-Akt signaling pathway, AGE-rage signaling pathway, and TNF signaling pathway. Therefore, using network pharmacology method and molecular docking technology to analyze the molecular biological network based on multi-target and multi-pathway, not only helps to deepen the understanding of AstragalusScorpion for PCa, but also provides the pharmacological basis for further research.

\section{Declarations}

\section{Acknowledgements}

Not applicable

\section{Authors' contributions}

Litong Wu and Xujun You conceived and designed the study. Ying Chen, Yueqin Yang and Minjing Chen collected the data. Zuzhao Che, Qixin Li and Wei Fu performed the data analysis, Litong Wu and Xujun You wrote the manuscript. All authors are responsible for reviewing data. All authors read and approved the final manuscript.

\section{Funding}

This work is financially supported by the Bao'an Traditional Chinese Medicine Development Foundation Research Program (Program No.2020KJCX-KTYJ-17), Bao'an District Science and Technology Innovation Bureau Research Program (Program No.2020JD536), Innovative project for doctoral students of Hunan University of Traditional Chinese Medicine (Program No.2020CX22) and Shenzhen Bao'an Traditional Chinese Medicine Hospital Research Program (Program No.BAZYY20200610). 
Availability of data and material

The data used to support the findings of this study are available from the corresponding author upon request, unless there are legal or ethical reasons for not doing so.

\section{Ethics approval and consent to participate}

Not applicable

\section{Consent for publication}

Not applicable

\section{Competing interests}

The authors declare that there are no conflicts of interest in relation to this work.

\section{References}

[1] Bray F, Ferlay J, Soerjomataram I, et al. Global cancer statistics 2018: GLOBOCAN estimates of incidence and mortality worldwide for 36 cancers in 185 countries. CA Cancer J Clin. 2018 Nov;68(6):394424.

[2] Kimura T, Egawa S. Epidemiology of prostate cancer in Asian countries. Int J Urol. 2018 Jun;25(6):524531.

[3] Chen W, Zheng R, Baade PD, et al. Cancer statistics in China, 2015. CA Cancer J Clin. 2016 MarApr;66(2):115-32.

[4] Glina S, Rivero MA, Morales A,et al. Studies on prostatic cancer I. The effect of castration, of estrogen and of androgen injection on serum phosphatases in metastatic carcinoma of the prostate by Charles Huggins and Clarence V. Hodges. J Sex Med. 2010 Feb;7(2 Pt 1):640-4.

[5] Mizushima N, Komatsu M. Autophagy: renovation of cells and tissues. Cell. 2011 Nov 11;147(4):72841.

[6] Rebbeck TR. Prostate Cancer Genetics: Variation by Race, Ethnicity, and Geography. Semin Radiat Oncol. 2017 Jan;27(1):3-10.

[7] Patil N, Gaitonde K. Clinical Perspective of Prostate Cancer. Top Magn Reson Imaging. 2016 Jun;25(3):103-8.

[8] Liu J, Wang S, Zhang Y, et al. Traditional Chinese medicine and cancer: History, present situation, and development. Thorac Cancer. 2015 Sep;6(5):561-9. 
[9] Sheng S, Wang J, Wang L, et al. Network pharmacology analyses of the antithrombotic pharmacological mechanism of Fufang Xueshuantong Capsule with experimental support using disseminated intravascular coagulation rats. J Ethnopharmacol. 2014 Jul 3;154(3):735-44.

[10] Ru J, Li P, Wang J, et al. TCMSP: a database of systems pharmacology for drug discovery from herbal medicines. J Cheminform. 2014 Apr 16;6:13.

[11] Liu Z, Guo F, Wang Y, et al. BATMAN-TCM: a Bioinformatics Analysis Tool for Molecular mechANism of Traditional Chinese Medicine. Sci Rep. 2016 Feb 16;6:21146.

[12] Safran M, Solomon I, Shmueli O, et al. GeneCards 2002: towards a complete, object-oriented, human gene compendium. Bioinformatics. 2002 Nov;18(11):1542-3.

[13] Szklarczyk D, Franceschini A, Wyder S, et al. STRING v10: protein-protein interaction networks, integrated over the tree of life. Nucleic Acids Res. 2015 Jan;43(Database issue):D447-52.

[14] Lopes CT, Franz M, Kazi F, et al. Cytoscape Web: an interactive web-based network browser. Bioinformatics. 2010 Sep 15;26(18):2347-8.

[15] Morris GM, Huey R, Lindstrom W, et al. AutoDock4 and AutoDockTools4: Automated docking with selective receptor flexibility. J Comput Chem. 2009 Dec;30(16):2785-91.

[16] Auyeung KK, Han QB, Ko JK. Astragalus membranaceus: A Review of its Protection Against Inflammation and Gastrointestinal Cancers. Am J Chin Med. 2016;44(1):1-22.

[17] Zhao Q, Chen XY, Martin C. Scutellaria baicalensis, the golden herb from the garden of Chinese medicinal plants. Sci Bull (Beijing). 2016;61(18):1391-1398.

[18] Srairi-Abid N, Othman H, Aissaoui D, et al. Anti-tumoral effect of scorpion peptides: Emerging new cellular targets and signaling pathways. Cell Calcium. 2019 Jun;80:160-174.

[19] Hu W, Xiao Z. Formononetin induces apoptosis of human osteosarcoma cell line U2OS by regulating the expression of Bcl-2, Bax and MiR-375 in vitro and in vivo. Cell Physiol Biochem. 2015;37(3):933-9.

[20] Rauf A, Imran M, Khan IA, et al. Anticancer potential of quercetin: A comprehensive review. Phytother Res. 2018 Nov;32(11):2109-2130.

[21] Imran M, Salehi B, Sharifi-Rad J, et al. Kaempferol: A Key Emphasis to Its Anticancer Potential. Molecules. 2019 Jun 19;24(12):2277.

[22] Kim C, Lee SG, Yang WM, et al. Formononetin-induced oxidative stress abrogates the activation of STAT3/5 signaling axis and suppresses the tumor growth in multiple myeloma preclinical model. Cancer Lett. 2018 Sep 1;431:123-141. 
[23] Wang AL, Li Y, Zhao Q, et al. Formononetin inhibits colon carcinoma cell growth and invasion by microRNA-149-mediated EphB3 downregulation and inhibition of PI3K/AKT and STAT3 signaling pathways. Mol Med Rep. 2018 Jun;17(6):7721-7729.

[24] Zhang J, Liu L, Wang J, Ren B, et al. Formononetin, an isoflavone from Astragalus membranaceus inhibits proliferation and metastasis of ovarian cancer cells. J Ethnopharmacol. $2018 \mathrm{Jul}$ 15;221:91-99.

[25] Wu XY, Xu H, Wu ZF, et al. Formononetin, a novel FGFR2 inhibitor, potently inhibits angiogenesis and tumor growth in preclinical models. Oncotarget. 2015 Dec 29;6(42):44563-78.

[26] Park S, Bazer FW, Lim W, et al. The O-methylated isoflavone, formononetin, inhibits human ovarian cancer cell proliferation by sub G0/G1 cell phase arrest through PI3K/AKT and ERK1/2 inactivation. J Cell Biochem. 2018 Sep;119(9):7377-7387.

[27] Nie X, Zhou Y, Li X, et al. Calycosin down-regulates c-Met to suppress development of glioblastomas. J Biosci. 2019 Sep;44(4):96.

[28] Wang K, Jin S, Fan D, et al. Anti-proliferative activities of finasteride in benign prostate epithelial cells require stromal fibroblasts and c-Jun gene. PLoS One. 2017 Feb 14;12(2):e0172233.

[29] Bromberg J, Wang TC. Inflammation and cancer: IL-6 and STAT3 complete the link. Cancer Cell. 2009 Feb 3;15(2):79-80.

[30] Tanaka T, Narazaki M, Kishimoto T. IL-6 in inflammation, immunity, and disease. Cold Spring Harb Perspect Biol. 2014 Sep 4;6(10):a016295.

[31] Hodge DR, Hurt EM, Farrar WL. The role of IL-6 and STAT3 in inflammation and cancer. Eur J Cancer. 2005 Nov;41(16):2502-12.

[32] Hayashi T, Fujita K, Nojima S, et al. High-Fat Diet-Induced Inflammation Accelerates Prostate Cancer Growth via IL6 Signaling. Clin Cancer Res. 2018 Sep 1;24(17):4309-4318.

[33] Guo Y, Nie Q, MacLean AL, et al. Multiscale Modeling of Inflammation-Induced Tumorigenesis Reveals Competing Oncogenic and Oncoprotective Roles for Inflammation. Cancer Res. 2017 Nov 15;77(22):6429-6441.

[34] Song Y, Wang H, Pan Y, et al. Investigating the Multi-Target Pharmacological Mechanism of Hedyotis diffusa Willd Acting on Prostate Cancer: A Network Pharmacology Approach. Biomolecules. 2019 Oct 9;9(10):591 .

[35] Neurath MF, Finotto S. IL-6 signaling in autoimmunity, chronic inflammation and inflammationassociated cancer. Cytokine Growth Factor Rev. 2011 Apr;22(2):83-9. 
[36] Huang P, Han J, Hui L. MAPK signaling in inflammation-associated cancer development. Protein Cell. 2010 Mar;1(3):218-26.

[37] Zhou YY, Li Y, Jiang WQ, et al. MAPK/JNK signalling: a potential autophagy regulation pathway. Biosci Rep. 2015 Apr 22;35(3):e00199.

[38] Lee S, Rauch J, Kolch W. Targeting MAPK Signaling in Cancer: Mechanisms of Drug Resistance and Sensitivity. Int J Mol Sci. 2020 Feb 7;21(3):1102.

[39] Altomare DA, Wang HQ, Skele KL, et al. AKT and mTOR phosphorylation is frequently detected in ovarian cancer and can be targeted to disrupt ovarian tumor cell growth. Oncogene. $2004 \mathrm{Jul}$ 29;23(34):5853-7.

[40] Yan Y, Huang H. Interplay Among PI3K/AKT, PTEN/FOXO and AR Signaling in Prostate Cancer. Adv Exp Med Biol. 2019;1210:319-331.

[41] Koundouros N, Poulogiannis G. Phosphoinositide 3-Kinase/Akt Signaling and Redox Metabolism in Cancer. Front Oncol. 2018 May 15;8:160.

[42] Karin M, Greten FR. NF-kappaB: linking inflammation and immunity to cancer development and progression. Nat Rev Immunol. 2005 Oct;5(10):749-59.

[43] Taniguchi K, Karin M. NF-KB, inflammation, immunity and cancer: coming of age. Nat Rev Immunol. 2018 May;18(5):309-324.

[44] Sun SC. The non-canonical NF-kB pathway in immunity and inflammation. Nat Rev Immunol. 2017 Sep;17(9):545-558.

[45] He T, Shen H, Wang S, et al. MicroRNA-3613-5p Promotes Lung Adenocarcinoma Cell Proliferation through a RELA and AKT/MAPK Positive Feedback Loop. Mol Ther Nucleic Acids. 2020 Sep 26;22:572583.

[46] Hua H, Kong Q, Zhang H, et al. Targeting mTOR for cancer therapy. J Hematol Oncol. 2019 Jul 5;12(1):71.

[47] Katsuya H, Cook LBM, Rowan AG, et al. Phosphatidylinositol 3-kinase- $\delta$ (PI3K- $\delta$ ) is a potential therapeutic target in adult T-cell leukemia-lymphoma. Biomark Res. 2018 Jul 18;6:24.

[48] Fizazi K, Albiges L, Loriot Y,et al. ODM-201: a new-generation androgen receptor inhibitor in castration-resistant prostate cancer. Expert Rev Anticancer Ther. 2015;15(9):1007-17.

[49] Yan Y, Huang H. Interplay Among PI3K/AKT, PTEN/FOXO and AR Signaling in Prostate Cancer. Adv Exp Med Biol. 2019;1210:319-331. 
[50] Vlassara $\mathrm{H}$, Brownlee $\mathrm{M}$, Cerami A. Accumulation of diabetic rat peripheral nerve myelin by macrophages increases with the presence of advanced glycosylation endproducts. J Exp Med. 1984 Jul 1;160(1):197-207.

[51] Neeper M, Schmidt AM, Brett J, et al. Cloning and expression of a cell surface receptor for advanced glycosylation end products of proteins. J Biol Chem. 1992 Jul 25;267(21):14998-5004.

[52] Hermani A, Hess J, De Servi B, et al. Calcium-binding proteins S100A8 and S100A9 as novel diagnostic markers in human prostate cancer. Clin Cancer Res. 2005 Jul 15;11(14):5146-52.

[53] Elangovan I, Thirugnanam S, Chen A,et al. Targeting receptor for advanced glycation end products (RAGE) expression induces apoptosis and inhibits prostate tumor growth. Biochem Biophys Res Commun. 2012 Jan 27;417(4):1133-8.

[54] Tang CH, Keng YT, Liu JF. HMGB-1 induces cell motility and a5 $\beta 1$ integrin expression in human chondrosarcoma cells. Cancer Lett. 2012 Sep 1;322(1):98-106.

[55] Li B, Rui J, Ding X, et al. Exploring the multicomponent synergy mechanism of Banxia Xiexin Decoction on irritable bowel syndrome by a systems pharmacology strategy. J Ethnopharmacol. 2019 Apr 6;233:158-168.

\section{Figures}




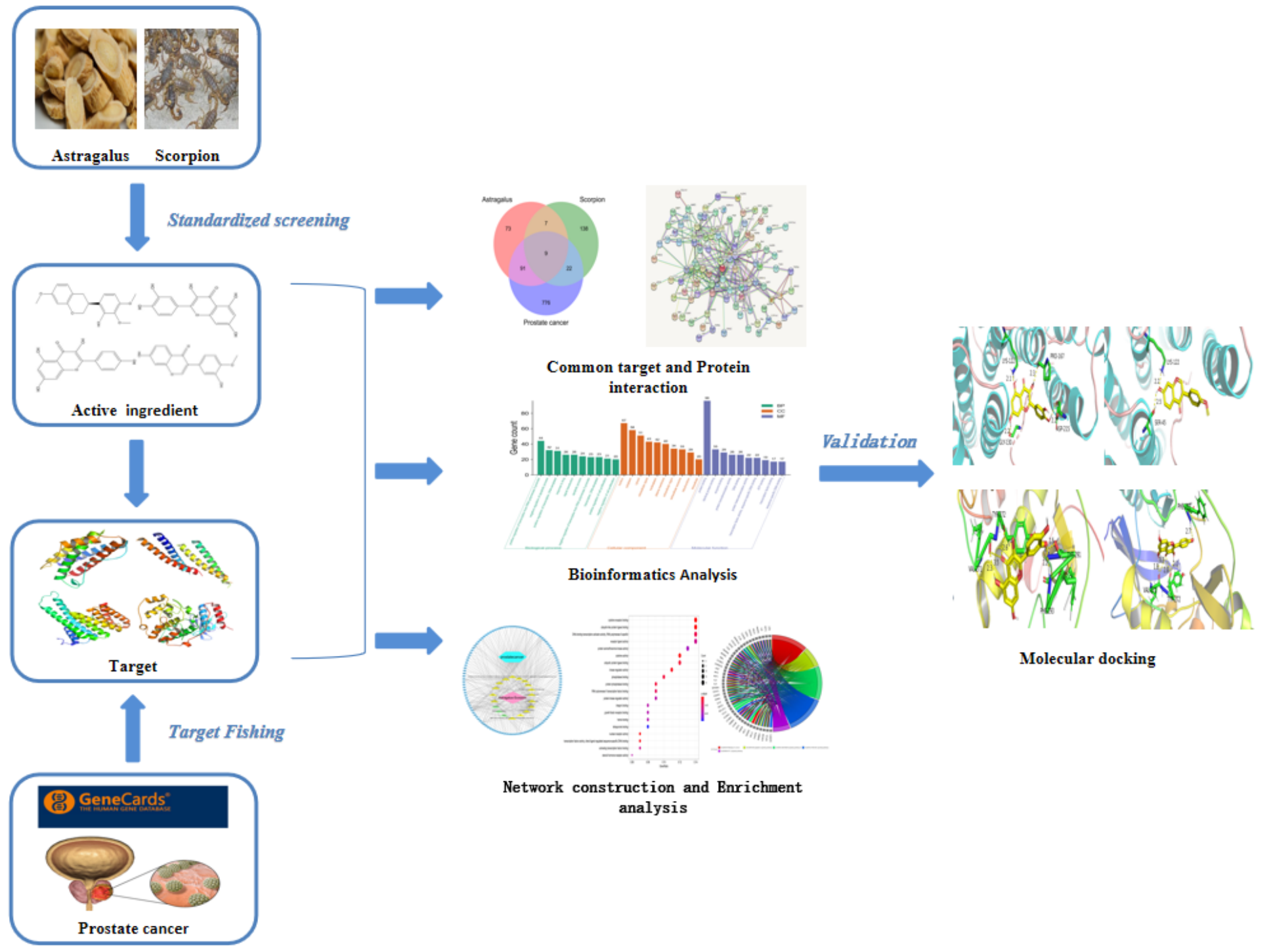

Figure 1

Network pharmacology analysis of Astragalus-Scorpion drug pair on prostate cancer 

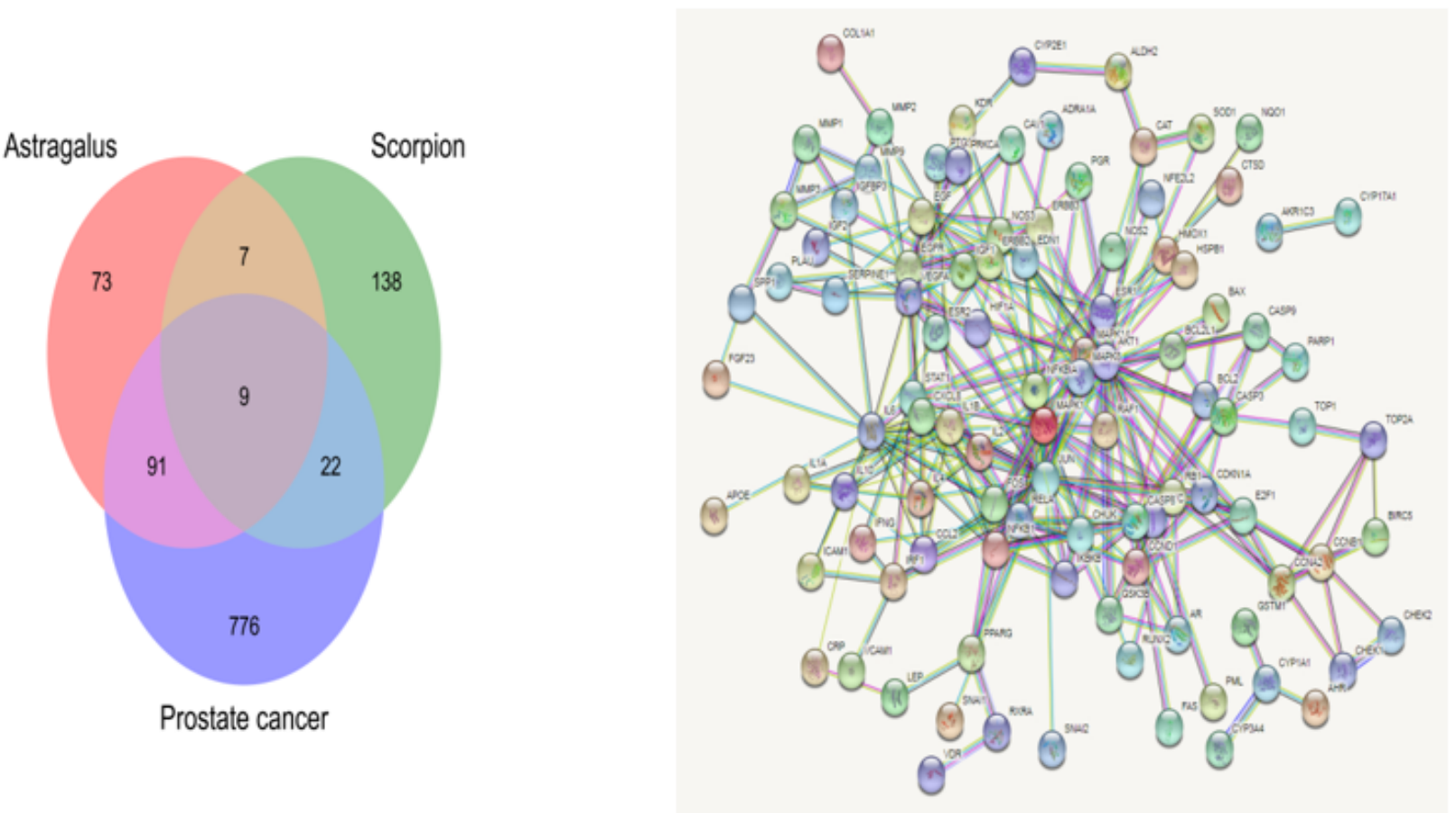

c

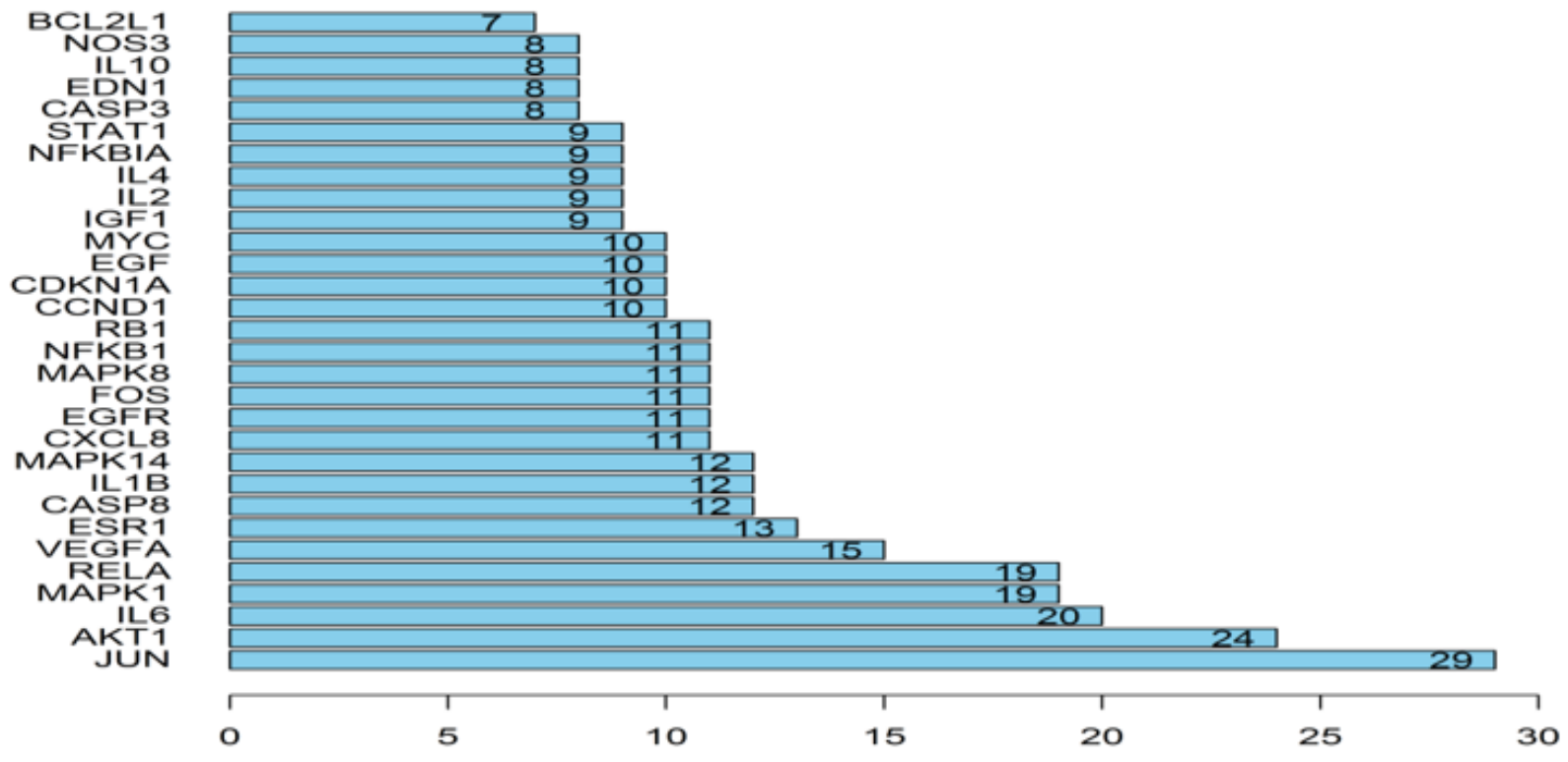

Figure 2

a. common target of Astragalus-Scorpion active ingredient target and potential target of PCa ; b. Protein interaction network of Astragalus -Scorpion in the treatment of PCa; c. Frequency display of proteinprotein interaction in the treatment of PCa with Astragalus -Scorpion drug pair. 


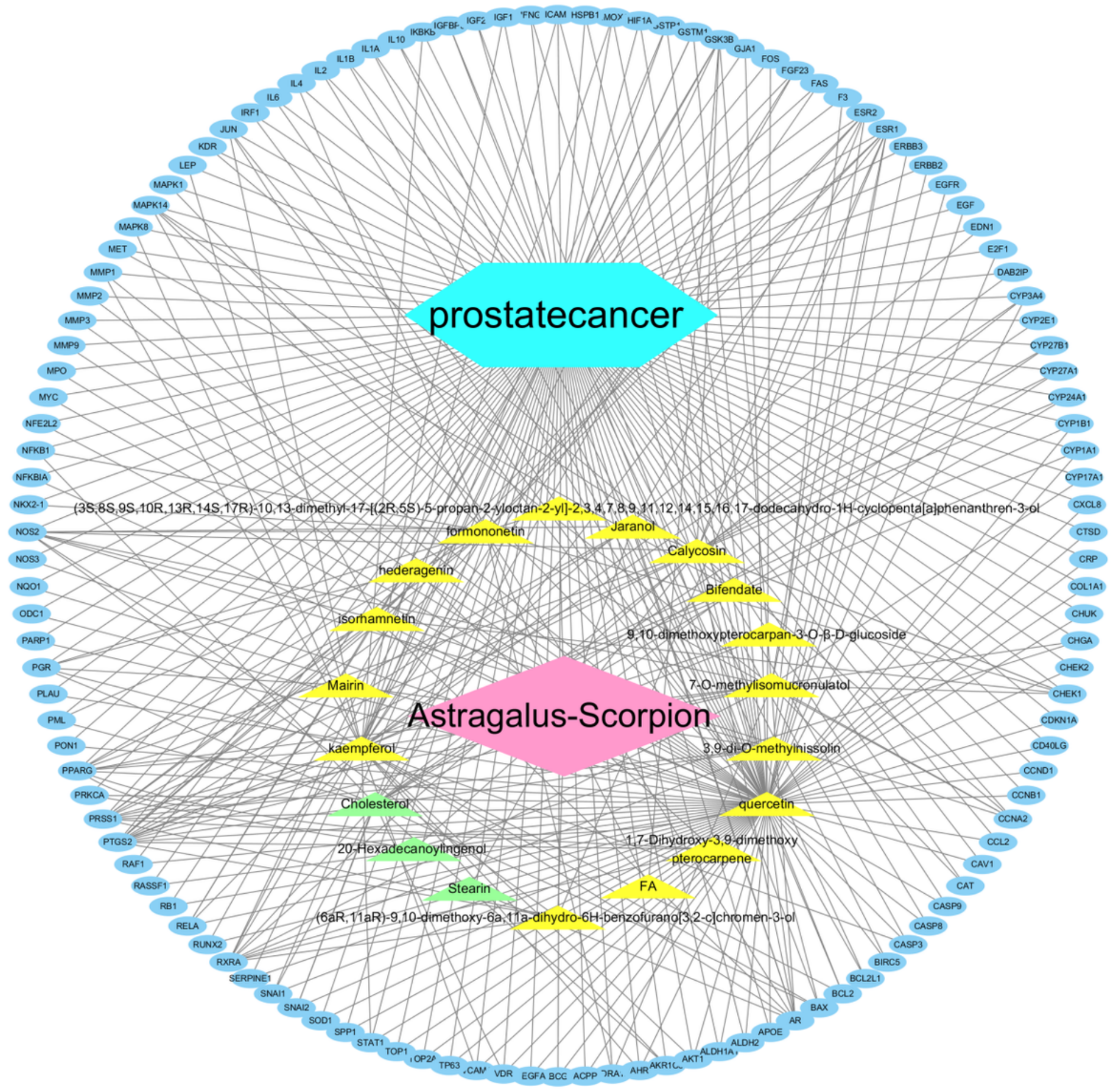

Figure 3

Active ingredient - target - disease network of Astragalus - Scorpion in the treatment of prostate cancer 


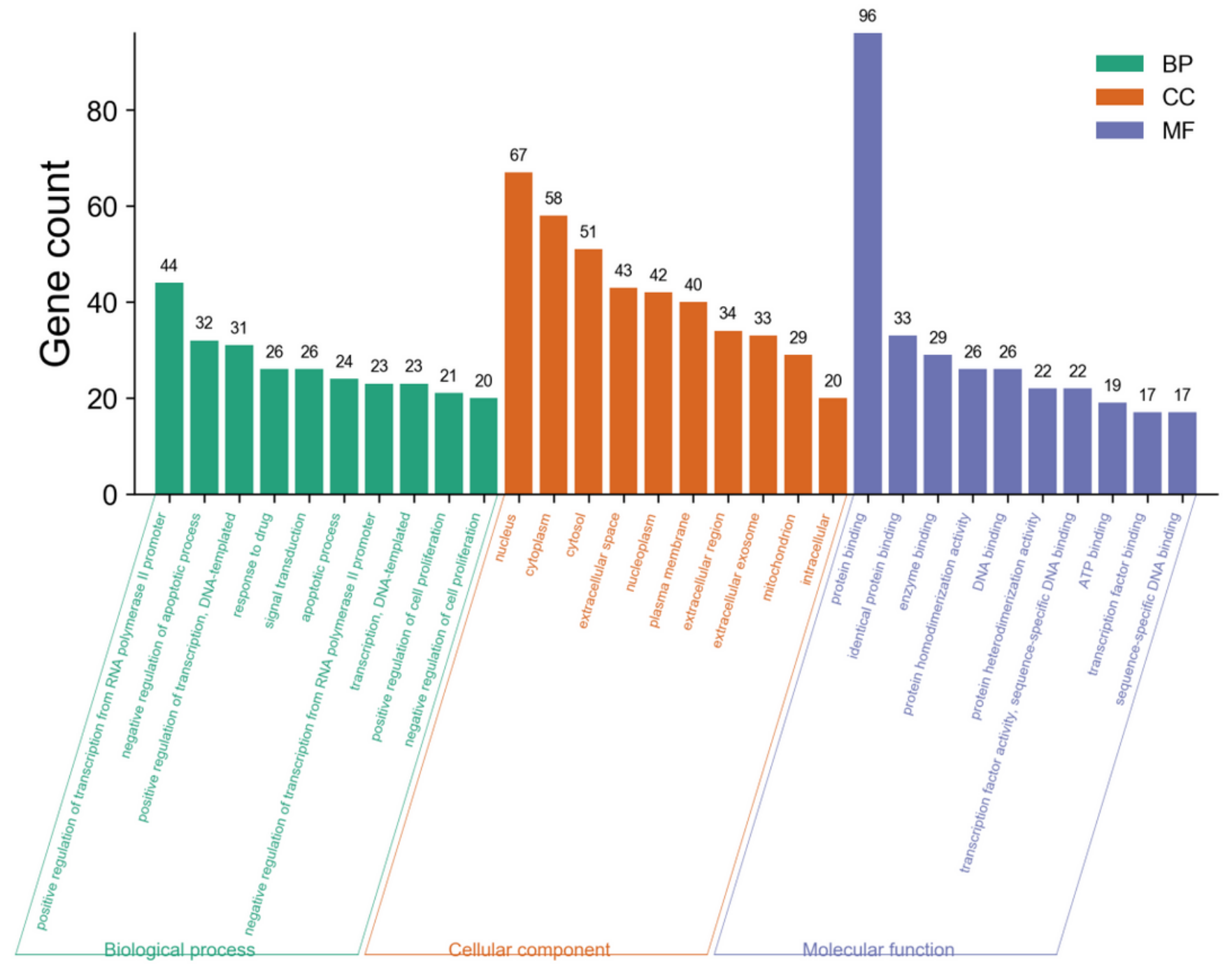

Figure 4

GO analysis of Astragalus-Scorpion drug pair for prostate cancer 
$\mathbf{a}$

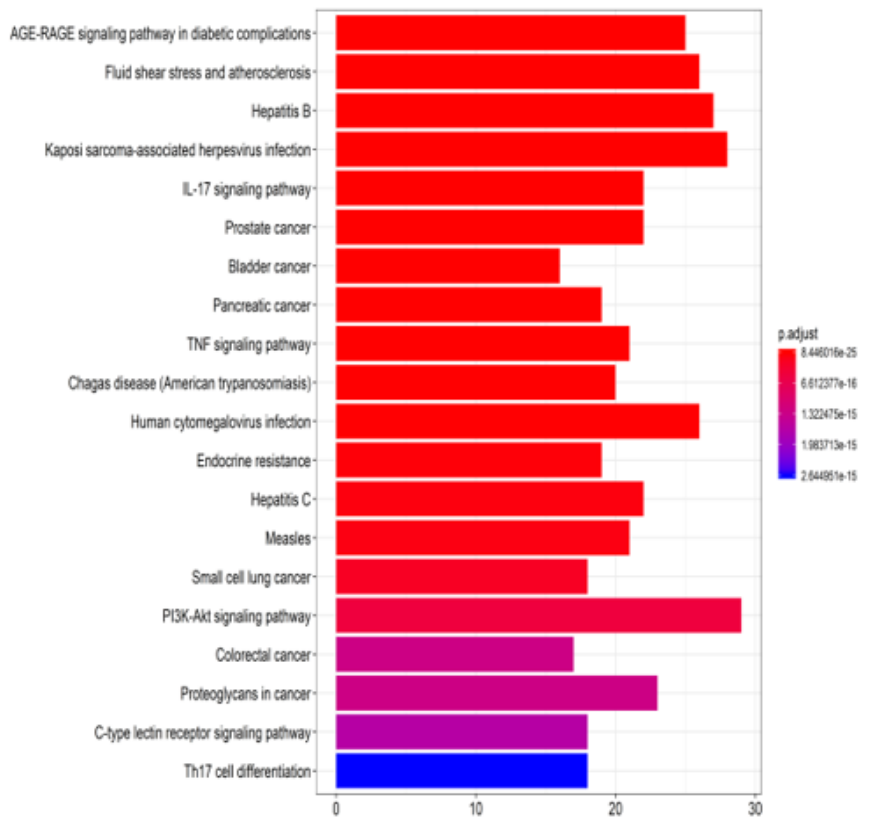

b

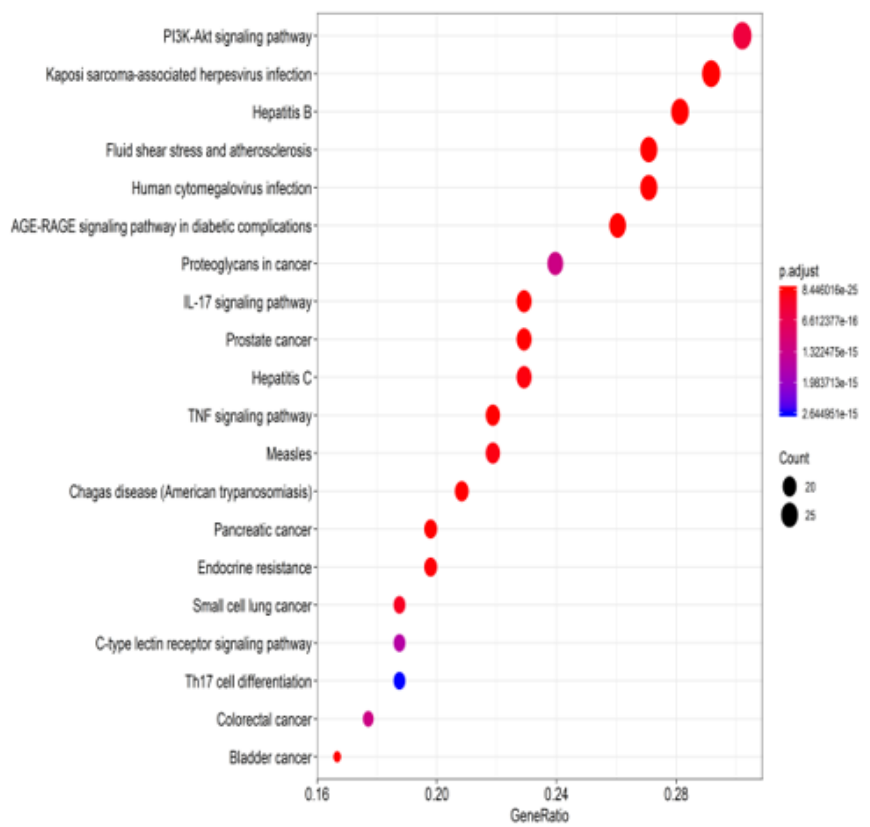

\section{Figure 5}

a. histogram of KEGG enrichment analysis of Astragalus-Scorpion drug pair for PCa; b. bubble diagram of KEGG enrichment analysis of Astragalus-Scorpion drug pair for $\mathrm{PCa}$ 


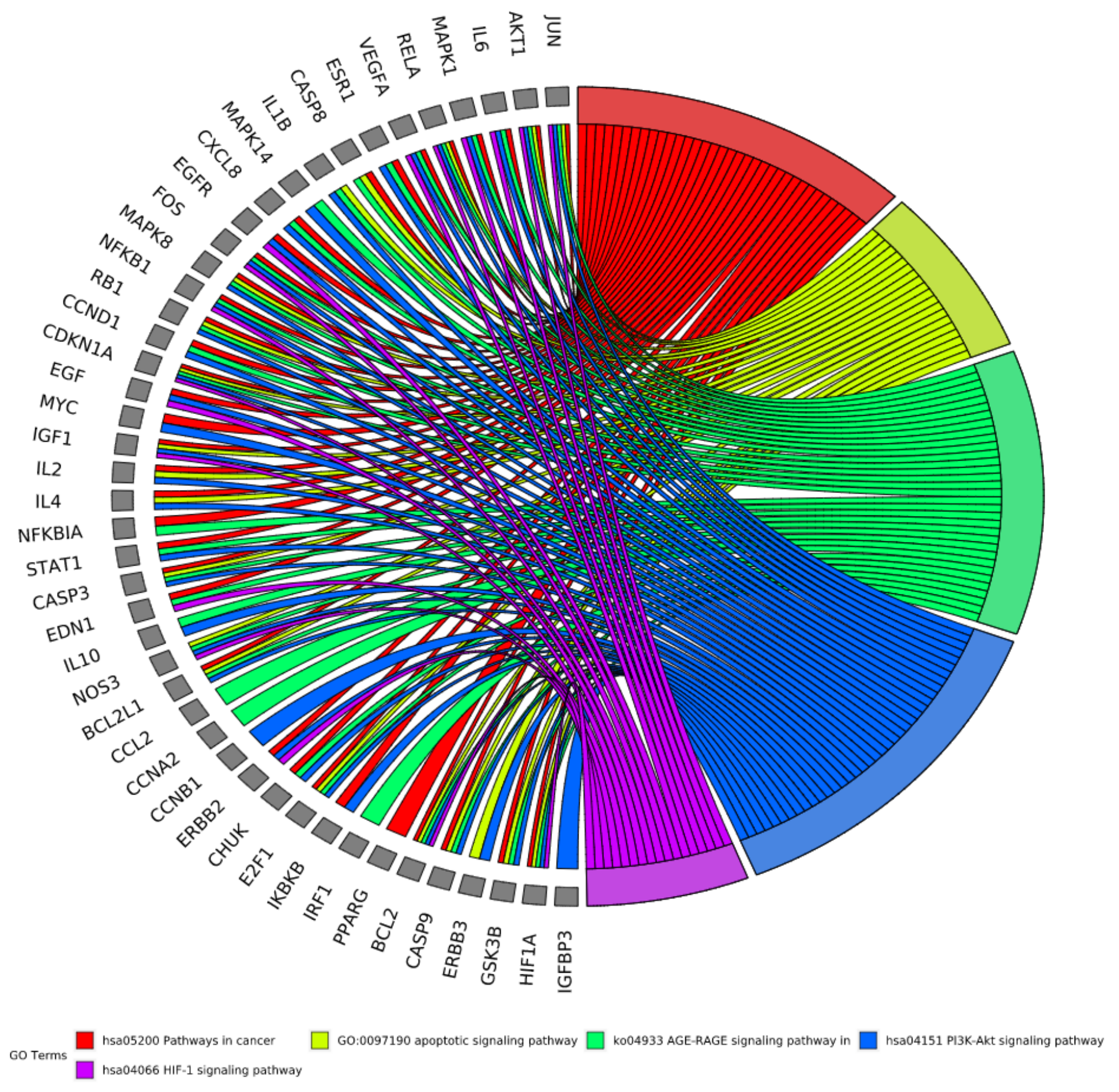

Figure 6

The KEGG enrichment analysis string diagram of the top 45 common targets. The different colors on the right of the diagram represent different signaling pathways, and on the left are the associated target genes. The more lines in the pathway,the more genes are enriched. 


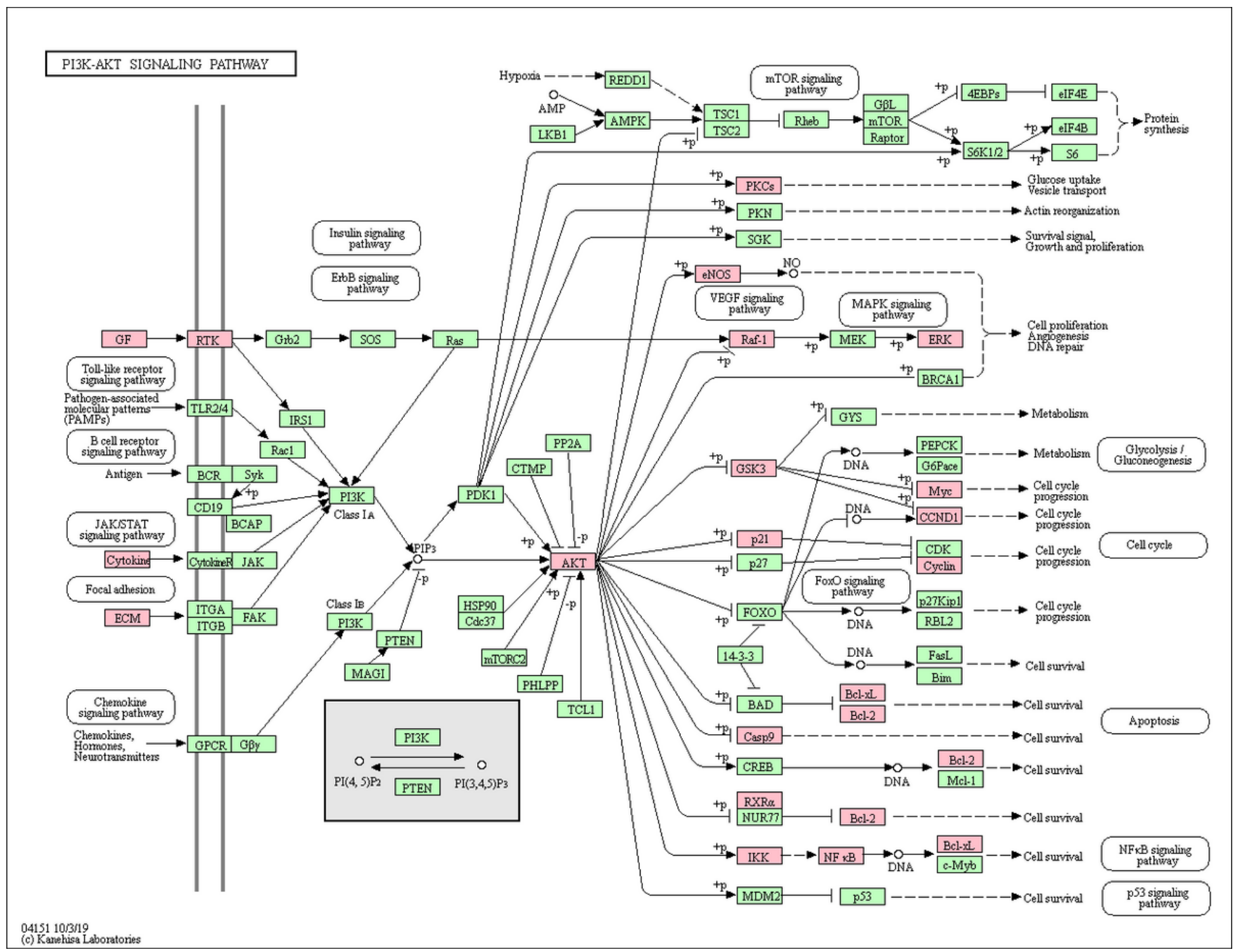

Figure 7

Potential targets and mechanism of Astragalus-Scorpion drug pair act on PI3K-Akt signaling pathway Note. The pink nodes represent common targets and their associated enzymes and compounds. 
a

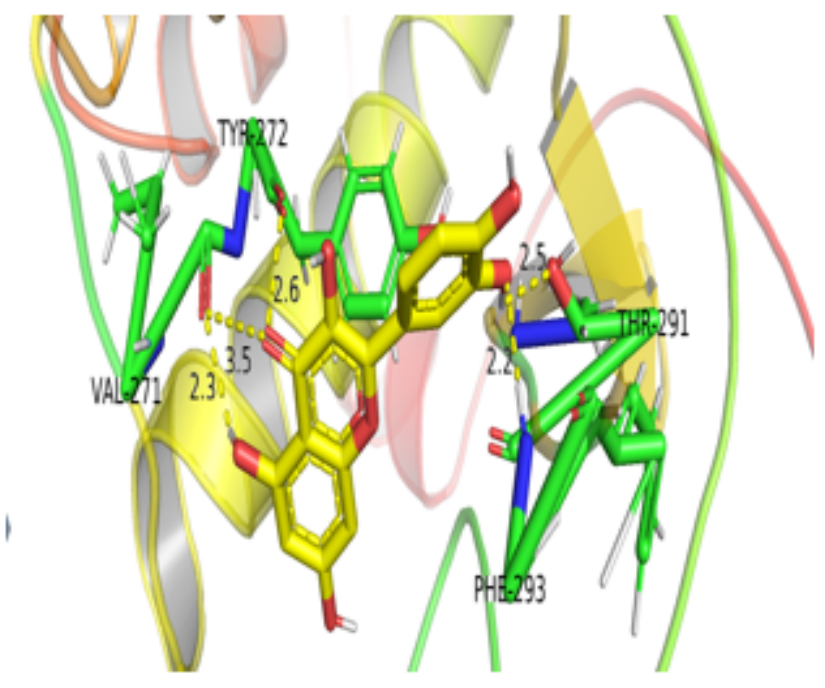

c

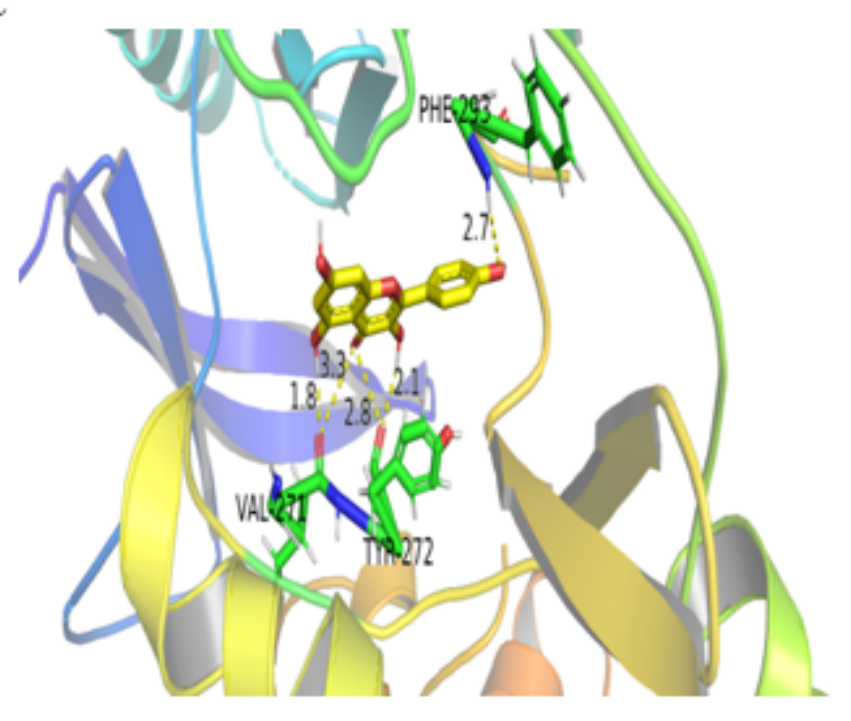

b
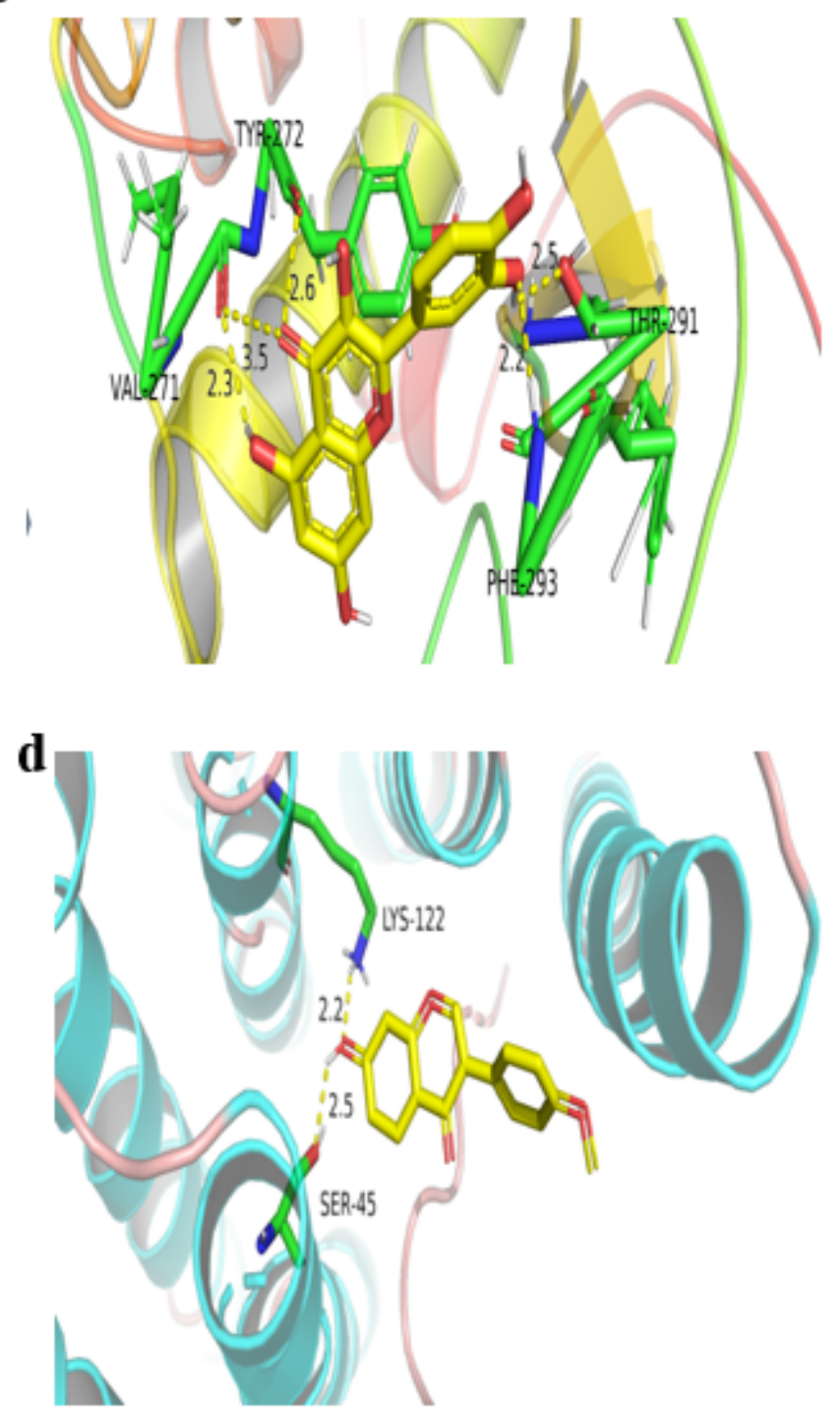

Figure 8

Molecular docking pattern diagram of active ingredient-target a.quercetin and AKT1; b.quercetin and RELA; c.kaempferol and AKT1; d.formononetin and RELA 